\title{
Isospin properties of the pasta phase with an extended statistical model from microscopic energy functionals
}

\author{
N. Alam, ${ }^{1, *}$ G. Chaudhuri, ${ }^{1,2, \dagger}$ and F. Gulminelli $\oplus^{3, *}$ \\ ${ }^{1}$ Physics Group, Variable Energy Cyclotron Centre, 1/AF Bidhan Nagar, Kolkata 700064, India \\ ${ }^{2}$ Homi Bhabha National Institute, Training School Complex, Anushakti Nagar, Mumbai 400085, India \\ ${ }^{3}$ Normandie University, ENSICAEN, UNICAEN, CNRS/IN2P3, LPC Caen, F-14000 Caen, France
}

(Received 18 June 2020; revised 6 October 2020; accepted 1 December 2020; published 22 December 2020)

\begin{abstract}
In this work, we have developed an extended statistical model to study nuclear multifragmentation reactions at intermediate energies, and we link the associated observables to the properties of supernova matter. The canonical thermodynamical model, used for this study, is modified by including inputs from the relativistic mean-field energy functionals. Even though the length scale of the supernova matter is very large compared to that of the multifragmentation reaction, we find an isospin observable, the average $\langle Z / N\rangle$ of the fragments, which is almost independent of the size of system, and can be directly compared to the estimation of fractionation in infinite nuclear matter. The isospin ratio $\langle Z / N\rangle$ of the heaviest cluster produced in nuclear fragmentation and of the corresponding pasta structure occurring in supernova matter are significantly lower than the spinodal estimation in uncharged nuclear matter, due to the presence of lighter isospin symmetric clusters that dominate the mass fraction in full equilibrium at finite temperature. The screening effect of electrons is also studied.
\end{abstract}

DOI: 10.1103/PhysRevC.102.064620

\section{INTRODUCTION}

When a massive star has exhausted all its nuclear fuel, it undergoes a huge explosion known as core-collapse supernova. Stellar matter in supernovae experience most extreme pressure and temperature conditions. During the supernova collapse the matter can reach temperatures (0.5-30) $\mathrm{MeV}$ and density $\sim\left(10^{-10}-3\right) \rho_{\text {sat }}$, where $\rho_{\text {sat }} \approx 0.15 \mathrm{fm}^{-3}$ is the saturation density of symmetric matter [1]. Such matter includes different phases and the phase transitions between them. In particular, in the outer layers of the exploding star, the thermodynamic conditions are such that strong (but not weak) equilibrium is established and matter is clusterized. To describe subsaturation supernova and early proto-neutron star matter, in the recent years, a number of improved equation of state $(\mathrm{EoS})$ models has been proposed that include the full nuclear distribution [2-14], but only a few of them have been used to produce tables which are publicly available [15]. Exploring the effect of the different microscopic ingredients and their uncertainties is not always simple in these models, because the impact of the energy functional can be hardly decoupled from the many-body description of the clusters, which is not the same in the different models. Some relevant properties of subsaturation warm supernova matter can be studied with simpler mean-field-based models including the full nuclear distribution only for the most abundant very light

\footnotetext{
*naosadphy@gmail.com

†'gargi@vecc.gov.in

†gulminelli@lpccaen.in2p3.fr
}

clusters [16-20] and the effect of the heavy clusters in the socalled single nucleus approximation [21]. In this picture, also known as "coexisting phase approximation," the composition of subsaturation matter is given by the coexistence of a dilute phase essentially composed of free nucleons, and a dense phase, given by a single heavy cluster or "pasta structure"; the finite size of the latter is determined by the competition between the surface and Coulomb energy. These studies have outlined the fact that, to reach a reasonably model independent description of supernova matter, inputs are needed from experimental data, and this especially concerns the surface energy [22] and the in-medium modifications of the binding energy in the medium [23,24]. This is, at least in principle, possible since similar thermodynamic conditions as in supernova matter can also be obtained in nuclear collisions studied in terrestrial nuclear experiments, especially the intermediate energy heavy ion collisions [4,20,23,25-27].

For this reason, it can be useful to have a unique statistical model which is able to describe both dilute supernova matter and fragmentation experiments within a same formalism [28]. There are, in general, two types of models that describe intermediate energy heavy ion collisions: (1) Dynamical models, such as, Boltzmann-Uehling-Uhlenbeck (BUU) model [29,30], antisymmetrized molecular dynamics (AMD) model [31], isospin-dependent quantum molecular dynamics (IQMD) model [32] and (2) statistical models, for example, canonical thermodynamical model (CTM) [33-36], statistical multifragmentation model (SMM) [37], microcanonical model [38], grand canonical model [34-36,39]. Though in principle it is possible to use the dynamical models also for supernova matter [40-42], statistical models clearly provide an 
easier application to the two different environments, provided equilibrated sources are carefully selected in the experimental data.

The two main difficulties in transposing the results of fragmentation experiments analyzed via statistical models to supernova matter are (1) the fact that the former are strongly affected by conservation laws [36], and (2) the presence of the neutralizing electron background in supernova matter, which modifies the Coulomb properties of the system [23]. These points can only be dealt theoretically, by considering the different environments in the unified statistical models, and compute the associated bias. The inclusion of an electron background is straightforward and can be done within the socalled Wigner-Seitz approximation. To account for finite size effects, in this work, we will use the canonical thermodynamical model (CTM) which includes the conservation laws effects linked to the finite number of particles in the experimental data. The CTM model has been modified by including the nuclear interactions from microscopic theory. In doing so, we built a new nuclear energy-density functional for finite nuclei to calculate the free energy of the clusters. The bulk contribution of this new functional is obtained using relativistic mean-field (RMF) approach [43-50]. The surface part of the energy-density functional is optimized using the experimental masses of spherical nuclei. With this extended CTM model, we can study the fragment yields and isotopic distributions of hot nuclear fragments produced in multifragmentation reactions. By increasing the size of the system towards the thermodynamic limit, we can check which observables are only marginally affected by finite size effects and can be used to give quantitative predictions for astrophysical observables, as well as to pin down the EoS dependence. This latter is analyzed employing two different parametrizations of the RMF model having large difference in the symmetry energy at saturation density. Finally, to transpose the results to supernova matter, the CTM model has been additionally modified by including electrons in the system. To this aim, we consider clusters as embedded in Wigner-Seitz cell to calculate the energy difference due to the presence of electrons in the system.

For this first application, we analyze the average isospin observable $\langle Z / N\rangle$ of the clusters produced in finite charged systems ( heavy ion collisions) as well as in infinite neutralized systems (supernova matter). This observable is chosen because it fulfills the requirements mentioned above, that is (1) it has negligible finite size effects, (2) it depends considerably on the symmetry energy. Moreover, this observable can be directly compared with the spinodal instability direction of infinite nuclear matter, which was often used in the literature to estimate the properties of the crust-core transition in neutron stars [51-55]. This comparison will allow highlighting the differences between spinodal decomposition occurring in metastable homogeneous matter, with respect to statistical equilibrium distributions that are believed to be explored both in fragmentation experiments and supernova matter. In particular, the charge content of the heavy clusters which are present at equilibrium in the surface of hot protoneutron stars is important to determine their thermal evolution [56-58].
This paper is organized as follows: In Sec. II, we give a brief summary of the CTM formalism and the RMF approach for calculation of nuclear density functional. In Sec. III, we present the main results and also try to shed light on the isospin properties of the clusters produced in the low density warm nuclear matter. Finally, the conclusions are drawn in Sec. IV.

\section{THEORETICAL FRAMEWORK}

\section{A. Canonical thermodynamical model}

The canonical thermodynamical model was introduced by Das Gupta and Mekjian more than 20 years ago [59], and it is described in details in many subsequent publications [33]. Here, we only report the basic equations needed for the present study.

The partition function in the canonical approach for a system of $A_{0}$ nucleons with $N_{0}$ neutrons and $Z_{0}$ protons inside a volume $V$ at a given temperature T can be written as

$$
Q_{N_{0}, Z_{0}}=\sum_{\left\{n_{N, Z}\right\}} \prod_{N, Z} \frac{\omega_{N, Z}^{n_{N, Z}}}{n_{N, Z} !} .
$$

Here, $\omega_{N, Z}$ represents the partition function of the composite with $N$ neutrons and $Z$ protons, and the sum is over all possible break-up channels, satisfying the conservation equations $N_{0}=\sum N n_{N, Z}$ and $Z_{0}=\sum Z n_{N, Z}$, where $N_{0}\left(Z_{0}\right)$ is the total neutron (proton) number in the system, and $n_{N Z}$ is the multiplicity of the $(N, Z)$ composite. The canonical partition function $Q_{N_{0}, Z_{0}}$ can be easily calculated using a recursion relation [33]:

$$
Q_{N_{0}, Z_{0}}=\frac{1}{Z_{0}} \sum_{N, Z} Z \omega_{N, Z} Q_{N_{0}-N, Z_{0}-Z} .
$$

Using the recursion relation, the average number of composites is given by

$$
n_{N, Z}=\omega_{N, Z} \frac{Q_{N_{0}-N, Z_{0}-Z}}{Q_{N_{0}, Z_{0}}}
$$

To compute $Q_{N_{0}, Z_{0}}$, we consider all nuclei $(N, Z)$ within the boundaries of neutron and proton drip lines. The partition function of a composite having $N$ neutrons and $Z$ protons is a product of two parts: one is due to the translational motion and the other is the intrinsic partition function of the composite:

$$
\omega_{N, Z}=\frac{V_{f}}{h^{3}}(2 \pi m T)^{3 / 2} A^{3 / 2} \times z_{N, Z}^{\text {int }},
$$

where $A=N+Z$ is the mass number of the composite and $V_{f}$ is the volume available for translational motion. Note that $V_{f}$ will be less than the total volume $V$, because a fraction of the volume is excluded by the presence of the other composites. We use $V_{f}=V-V_{0}$, where the excluded volume $V_{0}$ is given by

$$
V_{0}=\sum_{N, Z} n_{N, Z} \frac{A}{\rho_{0}(N, Z)},
$$

and $\rho_{0}$ is the cluster density. This latter is in principle expected to be isospin dependent [see Eq. (8) below], which induces 
a self-consistency in the resolution of Eq. (4). In the present work, following the standard version of the model [33], the approximation is made in Eq. $(5) \rho_{0}(N, Z) \approx \rho_{\text {sat }}$, where $\rho_{\text {sat }}$ is the saturation density of symmetric nuclear matter taken as $\rho_{\text {sat }}=0.148 \mathrm{fm}^{-3}$ for the models considered in this paper. This leads to the simple expression $V_{0}=A_{0} / \rho_{\text {sat }}$, thus avoiding the self-consistency. For nuclei in isolation, the internal partition function is given by $z_{N, Z}^{\text {int }}=\exp [-\beta F(N, Z)]$, where $F$ is the free energy of the composite, to be specified below.

\section{B. Energy calculation for the composites}

The intrinsic free energy of the composites at freeze-out can be approximated as the sum of a bulk and a surface term:

$$
F=F_{\text {bulk }}(A, I, T)+F_{\text {surf }}(A, I, T),
$$

with $I=(N-Z) / A$. Isolating the ground-state energy in the bulk term leads to:

$$
\begin{aligned}
F_{\text {bulk }}= & e\left(\rho_{0}(I), I\right) A+E_{\text {coul }}(A, Z) \\
& +E^{*}(A, T)-T S(A, T) .
\end{aligned}
$$

In this equation, the first two terms on the right-hand side represent the bulk nuclear and coulomb contributions to the ground-state energy, while the third and fourth term represent the bulk part of the excitation energy and entropy, respectively.

Finite size effects are introduced via the surface free energy $F_{\text {surf }}(A, I, T)=\sigma_{\text {surf }}(I, T) A^{2 / 3}$, with $\sigma_{\text {surf }}(I, T)$ the isospin dependent finite temperature surface tension. The different parameters entering Eqs. (6) and (7) are detailed below.

The nuclear bulk term $e\left(\rho_{0}, I\right)$ represents the energy per nucleon of bulk nuclear matter at $T=0$, isospin asymmetry $I=\left(\rho_{0 n}-\rho_{0 p}\right) / \rho_{0} \equiv(N-Z) / A$, and total density $\rho_{0}=$ $\rho_{0 n}+\rho_{0 p}$, where $\rho_{0}(I)$ is the saturation density of asymmetric nuclear matter corresponding to asymmetry $I$. This latter depends on the empirical nuclear parameters, $L_{\mathrm{sym}}, K_{\mathrm{sym}}$, and $K_{\text {sat }}$ as [60]

$$
\rho_{0}(I)=\rho_{\mathrm{sat}}\left[1-\frac{3 L_{\mathrm{sym}} I^{2}}{K_{\mathrm{sat}}+K_{\mathrm{sym}} I^{2}}\right] .
$$

The energy per nucleon of bulk nuclear matter $e(\rho, I)$, as well as the associated empirical parameters $\rho_{\text {sat }}$, $K_{\text {sat }}=9 \rho_{\text {sat }}^{2} \partial^{2} e /\left.\partial \rho^{2}\right|_{\rho_{\text {sat }}}, \quad L_{\text {sym }}=3 \rho_{\text {sat }} d e_{\text {sym }} /\left.d \rho\right|_{\rho_{\text {sat }}} \quad$ and $K_{\text {sym }}=9 \rho_{\text {sat }}^{2} d^{2} e_{\text {sym }} /\left.d \rho^{2}\right|_{\rho_{\text {sat }}}$, have been calculated in the framework of the RMF approach, as detailed in Sec. II C. In these expressions, the symmetry energy is introduced with the usual definition:

$$
e_{\mathrm{sym}}(\rho)=\left.\frac{\rho^{2}}{2} \frac{\partial^{2} e}{\partial(\rho I)^{2}}\right|_{I=0} .
$$

Considering spherical symmetry, the bulk Coulomb energy term is approximated as

$$
E_{\text {coul }}(A, Z)=\frac{3}{5} \frac{e^{2} Z^{2} \alpha}{r_{0} A^{1 / 3}},
$$

where $r_{0}=\left(3 /\left(4 \pi \rho_{0}(I)\right)\right)^{1 / 3}$.

In principle, the bulk excitation energy and entropy should also be consistently calculated from the same energy functional used for the ground-state quantities, within a finite temperature RMF calculation. However, in the mean-field theory, finite temperature does not affect the energy-density functional but only the occupation of single particle energies. As a consequence, the bulk excitation energy and entropy do not depend on the equation of state through its empirical parameters, but only on the effective mass and the mass splitting. On this issue, microscopic approaches such as BHF and DBHF consistently point towards a positive mass splitting $\left(m_{n}^{*}>m_{p}^{*}\right)$ in neutron rich matter $[61,62]$ However, the amplitude of the splitting and its density and isospin dependence are poorely known, and can be hardly constrained by terrestrial experiments and astrophysical observations [63]. No mass splitting is assumed in the functionals used in the present work, and the two functionals have the same effective mass. Because of the poor knowledge about the isospin dependence of the effective mass, and to highlight the effect of the empirical parameters, for this first application we have therefore neglected the functional dependence of $E^{*}$ and $S$ and used the same low temperature Sommerfeld expansion of the free Fermi gas approximation which was employed in previous applications of the CTM [33]:

$$
E^{*}=\frac{A \pi^{2}}{4 e_{F}} T^{2} ; \quad S=\frac{A \pi^{2}}{2 e_{F}} T,
$$

where $e_{F}$ is the Fermi energy at symmetric nuclear matter saturation. We expect that this approximation does not affect the qualitative results of this paper, which is essentially aimed at exploring the role of light clusters in the thermodynamic equilibrium. A fully consistent treatment will be however needed for a direct comparison of the different functionals with experimental data, and it is left for future work. An alternative interesting possibility will be to use phenomenological parametrizations for the level densities [64], which contain in an effective way beyond-mean-field corrections to the entropy term of finite nuclei.

For the calculation of the surface free energy at arbitrary proton fractions, we use the expression suggested from Thomas-Fermi calculations [65] which was later used in many calculations on neutron star and supernova modeling within the compressible liquid-drop model [21,66-68]. The temperature dependence is accounted for, by using the expression proposed in Ref. [33]. Curvature terms are neglected, and the complete parameterized form of the surface tension is

$$
\sigma_{\text {surf }}=4 \pi r_{0}^{2} \sigma_{0}\left[\frac{2^{p+1}+b}{\left(\frac{Z}{A}\right)^{-p}+b+\left(\frac{N}{A}\right)^{-p}}\right]\left[\frac{T_{c}^{2}-T^{2}}{T_{c}^{2}+T^{2}}\right]^{5 / 4}
$$

The parameter $\sigma_{0}$ physically corresponds to the surface tension at zero temperature and zero isospin asymmetry, while $b$ governs the isospin dependence for moderate asymmetries. Concerning the $p$ parameter, it governs the behavior at extreme asymmetries. This latter parameter cannot be fixed from observables of terrestrial nuclei and is characteristic of the symmetry energy properties of the different functionals [22].

The determination of those parameters for the different functionals will be explained in Sec. III.

In principle, also the critical temperature $T_{c}$ entering Eq. (12) is functional dependent [55]. However, the difference between the different functionals does not produce any 
measurable effect at the temperatures we will be interested in. We have therefore fixed $T_{c}=18 \mathrm{MeV}$.

\section{Bulk matter functional}

The RMF approach, a relativistic description of nuclear matter within a field theoretical framework, has been employed to obtain the bulk energy of the composites. In the conventional RMF theory [50] nucleons are treated as elementary particles and interactions between the nucleons are mediated by the exchange of scalar-isoscalar $\sigma$, vectorisoscalar $\omega$, and vector-isovector $\rho$ mesons. These $\sigma, \omega$, and $\rho$ mesons are described here by $\phi, V^{\mu}$, and $\mathbf{b}^{\mu}$ fields, respectively. In this theory, several self- and cross-interaction terms of the mesons are also used to yield the saturation properties correctly and vary the density dependence of symmetry energy. The Lagrangian density for the extended RMF model can be written as

$$
\mathcal{L}=\sum_{i=p, n} \mathcal{L}_{i}+\mathcal{L}_{\sigma}+\mathcal{L}_{\omega}+\mathcal{L}_{\rho}+\mathcal{L}_{\omega \rho},
$$

where the nucleon Lagrangian reads

$$
\mathcal{L}_{i}=\bar{\psi}_{i}\left[\gamma_{\mu} i D^{\mu}-M^{*}\right] \psi_{i}
$$

with

$$
\begin{aligned}
i D^{\mu} & =i \partial^{\mu}-g_{v} V^{\mu}-\frac{g_{\rho}}{2} \boldsymbol{\tau} \cdot \mathbf{b}^{\mu} \\
M^{*} & =M-g_{s} \phi .
\end{aligned}
$$

The mesonic Lagrangians are given by

$$
\begin{aligned}
& \mathcal{L}_{\sigma}=+\frac{1}{2}\left(\partial_{\mu} \phi \partial^{\mu} \phi-m_{s}^{2} \phi^{2}-\frac{1}{3} \kappa \phi^{3}-\frac{1}{12} \lambda \phi^{4}\right), \\
& \mathcal{L}_{\omega}=-\frac{1}{4} \Omega_{\mu \nu} \Omega^{\mu \nu}+\frac{1}{2} m_{v}^{2} V_{\mu} V^{\mu}+\frac{1}{4 !} \xi g_{v}^{4}\left(V_{\mu} V^{\mu}\right)^{2}, \\
& \mathcal{L}_{\rho}=-\frac{1}{4} \mathbf{B}_{\mu \nu} \cdot \mathbf{B}^{\mu \nu}+\frac{1}{2} m_{\rho}^{2} \mathbf{b}_{\mu} \cdot \mathbf{b}^{\mu},
\end{aligned}
$$

where $\quad \Omega_{\mu \nu}=\partial_{\mu} V_{\nu}-\partial_{\nu} V_{\mu}, \quad \mathbf{B}_{\mu \nu}=\partial_{\mu} \mathbf{b}_{v}-\partial_{\nu} \mathbf{b}_{\mu}-$ $g_{\rho}\left(\mathbf{b}_{\mu} \times \mathbf{b}_{v}\right)$, and $\boldsymbol{\tau}$ are the Pauli matrices. The mesonic Lagrangian is supplemented with the following nonlinear term that mix the $\omega$ and $\rho$ mesons,

$$
\mathcal{L}_{\omega \rho}=\Lambda_{v} g_{v}^{2} g_{\rho}^{2} \mathbf{b}_{\mu} \cdot \mathbf{b}^{\mu} V_{\mu} V^{\mu}
$$

This nonlinear $\omega-\rho$ mixing term in the RMF Lagrangian allows us to vary the density dependence of symmetry energy, hence to study the effects symmetry energy on cluster formation [69].

A set of field equations can be derived from the above Lagrangian density by using the Euler-Lagrange equation. These field equations are nonlinear coupled equations and very hard to solve exactly. However, these equations can be simplified and solved self-consistently by adopting mean-field approximation, i.e., the meson-field operators are replaced by their expectation values. The ground-state energy per baryon of the system as a function of nucleon number density $\rho$ and isospin asymmetry $I$ can be expressed in terms of model
TABLE I. EOS parameters calculated at saturation density for NL3 and NL3 $\omega \rho 6$ models.

\begin{tabular}{lccccccc}
\hline \hline Model & $\begin{array}{c}\rho_{0} \\
\left(\mathrm{fm}^{-3}\right)\end{array}$ & $\begin{array}{c}E_{0} \\
(\mathrm{MeV})\end{array}$ & $\begin{array}{c}K_{0} \\
(\mathrm{MeV})\end{array}$ & $\begin{array}{c}Q_{0} \\
(\mathrm{MeV})\end{array}$ & $\begin{array}{c}E_{\text {sym }} \\
(\mathrm{MeV})\end{array}$ & $\begin{array}{c}L_{\text {sym }} \\
(\mathrm{MeV})\end{array}$ & $\begin{array}{c}K_{\text {sym }} \\
(\mathrm{MeV})\end{array}$ \\
\hline NL3 & 0.148 & -16.25 & 271.6 & 205.5 & 37.4 & 118.5 & 100.9 \\
NL3 $\omega \rho 6$ & 0.148 & -16.25 & 271.6 & 205.5 & 31.7 & 55.3 & -7.5 \\
\hline \hline
\end{tabular}

parameters and mesonic mean fields as

$$
\begin{aligned}
e(\rho, I)= & {\left[\sum_{i=p, n} \frac{1}{\pi^{2}} \int_{0}^{k_{f}^{i}} d p p^{2} \sqrt{p^{2}+M^{* 2}}+g_{v} V_{0} \rho\right.} \\
& +\frac{g_{\rho}}{2} b_{0} \rho_{3}+\frac{1}{2}\left((\nabla \phi)^{2}+m_{s}^{2} \phi^{2}\right)+\frac{\kappa}{3 !} \phi^{3}+\frac{\lambda}{4 !} \phi^{4} \\
& -\frac{1}{2}\left(\left(\nabla V_{0}\right)^{2}+m_{v}^{2} V_{0}^{2}+\frac{\xi g_{v}^{4}}{12} V_{0}^{4}\right) \\
& \left.-\frac{1}{2}\left[\left(\nabla b_{0}\right)^{2}+m_{\rho}^{2} b_{0}^{2}\right]-\Lambda_{v} g_{v}^{2} g_{\rho}^{2} V_{0}^{2} b_{0}^{2}\right] / \rho-M,
\end{aligned}
$$

with nucleon effective mass $M^{*}=M-g_{s} \phi$.

The parameters of the models are the couplings of the mesons to the nucleons $g_{s}, g_{v}$, and $g_{\rho}$, the nucleon bare mass $M$, the meson masses, the self-interacting coupling constants, $\kappa, \lambda$, and $\xi$, and the coupling constants of the nonlinear mixing term, $\Lambda_{v}$. In the literature, these free parameters are determined by fits to experimental data, with the exception of $\Lambda_{v}$ that is tuned to explore different density dependencies for the symmetry energy. For our calculations, we use the parameter sets NL3 and NL3 $\omega \rho 6$ [55,70]. These two parameter sets only differ from one another by the strength of the coupling parameter $\Lambda_{v}$.

\section{RESULTS AND DISCUSSION}

For our study, we have employed two different versions of the RMF model, namely, NL3 and NL3 $\omega \rho 6$. The values of the isoscalar and isovector EoS parameters at the saturation density for these two models are listed in Table I. These two models have the same equation of state for symmetric nuclear matter, but their symmetry energy behavior is strongly different. Specifically, NL3 model has significantly stiffer symmetry energy compared to the NL3 $\omega \rho 6$. Such choice of the models allows us to examine the effects of symmetry energy on various observables obtained within the extended CTM calculation.

\section{A. Ground-state properties}

We first examine the difference between the ground-state properties of nuclei, as implied by the choice of the two different functionals, within the simple liquid drop treatment presented in Sec. II B.

This different behavior modifies the saturation properties of nuclear matter, and therefore the internal density of the fragments that are produced in the thermodynamic 

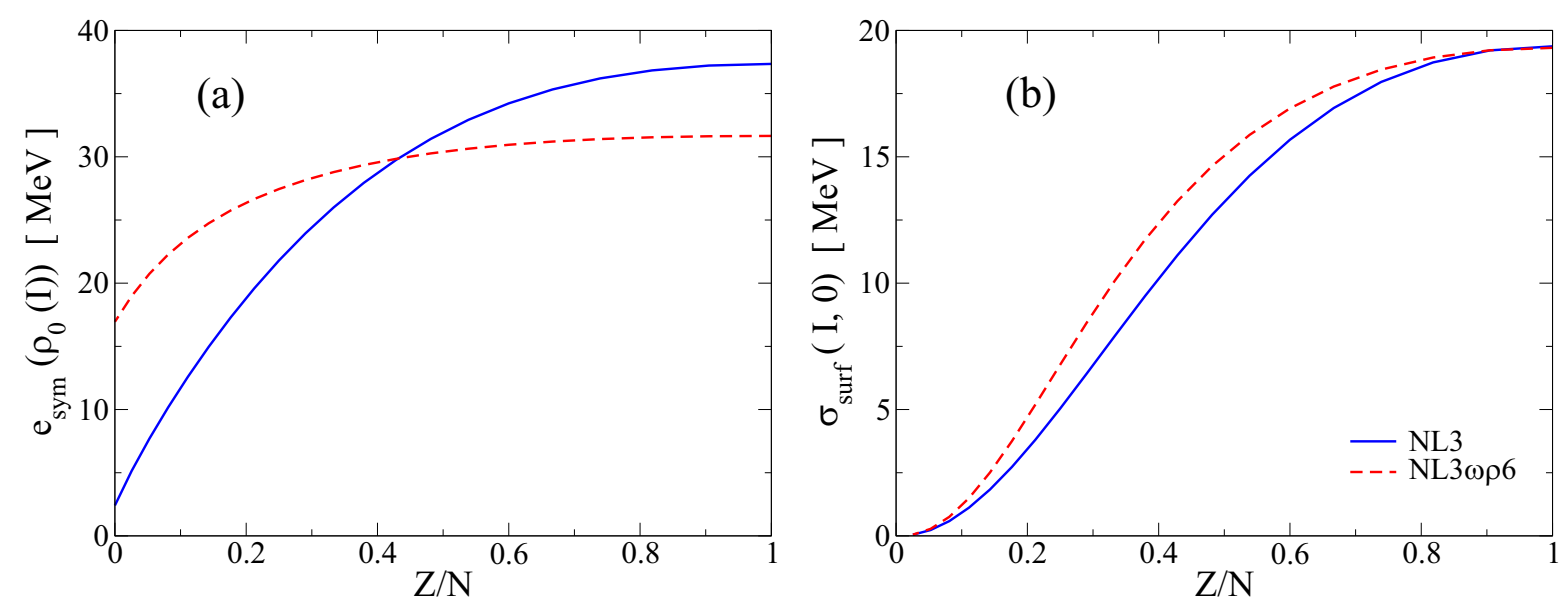

FIG. 1. Bulk symmetry energy per particle (left part) and ground-state surface tension (right part) as a function of the $Z / N$ ratio of the clusters for NL3 (solid blue) and NL3 $\omega \rho 6$ (dashed red) models.

equilibrium, as shown in the left part of Fig. 1. We can see that for moderate isospin asymmetries the symmetry energy is higher with the stiffer parameter set, while the opposite is true at high isospin values close to the neutron dripline. We can anticipate that in the case of the NL3 set, we will predict in very asymmetric systems a non negligible probability for exotic clusters, which correspond to an increased binding.

However, to understand the consequence of the symmetry energy properties on the matter composition, one needs to examine the behavior of the surface energy Eq. (12), which also depends on the adopted energy functional. In this equation, the parameters $\sigma_{0}$ and $b$ are determined by fitting the experimental binding energies of the spherical magic nuclei, $(40,20),(48,20),(48,28),(58,28),(88,38),(90,40),(114,50)$, $(132,50)$, and $(208,82)$. As already mentioned, the $p$ parameter governs the behavior at extreme asymmetries and it cannot be fixed from observables of terrestrial nuclei [22]. For a given functional (see Sec. IIC), we obtain the value of $p$ by imposing that at zero temperature the crust-core transition density calculated from the crust modeling with this same parameter set, using the technique of Ref. [22], is consistent with the value obtained from the dynamical spinodal analysis given in the literature [53,54]. For more details, see Ref. [22].

The resulting behavior of the surface tension for the two functionals used in this work is displayed in the right part of Fig. 1 as a function of the asymmetry, and the values of the different parameters are reported in Table II. We can see that the NL3 model corresponds to a lower surface tension for all isospin values. For nuclei of moderate asymmetries, the higher bulk energy of NL3 observed in the left part of

TABLE II. Surface parameters for the two chosen functionals obtained from the fitting of the nuclear masses along with the the crust-core transition density (see text).

\begin{tabular}{lccr}
\hline \hline Model & $p$ & $\sigma_{0}\left(\mathrm{MeV} \mathrm{fm}^{-2}\right)$ & \multicolumn{1}{c}{$b$} \\
\hline NL3 & 2.82 & $1.1208 \pm 0.0019$ & $3.9416 \pm 0.2312$ \\
NL3 $\omega \rho 6$ & 2.65 & $1.1173 \pm 0.0018$ & $12.9670 \pm 0.5046$ \\
\hline \hline
\end{tabular}

Fig. 1 is thus compensated by a lower surface energy. This can be understood from the fact that the global (bulk and surface) binding energy is constrained by the experimental data. However, at high I values, the lower symmetry energy of the stiffer functional NL3 corresponds to a surface energy which is also reduced, leading to a global higher binding in NL3 for very asymmetric nuclei. This is understood from the well known correlation between the $L_{\text {sym }}$ parameter and the transition density [22]: The very low transition density of NL3 implies a faster reduction of the surface energy as a function of the isospin.

An immediate consequence of these observations is the shift in the dripline between the two functionals. In Fig. 2 we present the neutron and proton driplines which are obtained using these models. We can see that NL3 and NL3 $\omega \rho 6$ have almost identical proton dripline, but different neutron dripline, as expected from their different symmetry energy properties discussed above. Specifically, the increased stability of neutron rich clusters in NL3 leads to a shift in the neutron drip line that can attain five units for the heavier isotopes. One could expect that an even increased effect could be observed

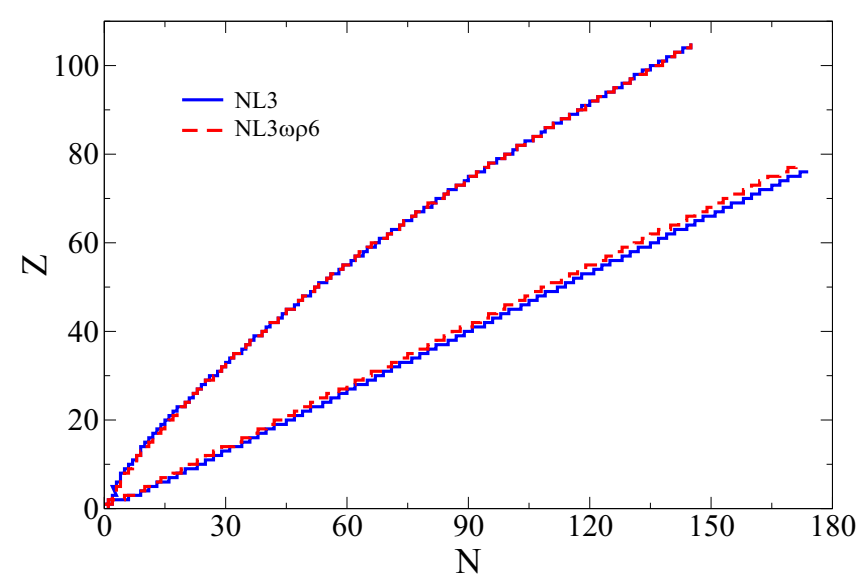

FIG. 2. Neutron and proton dripline for NL3 (solid blue) and NL3 $\omega \rho 6$ (dashed red) models. 


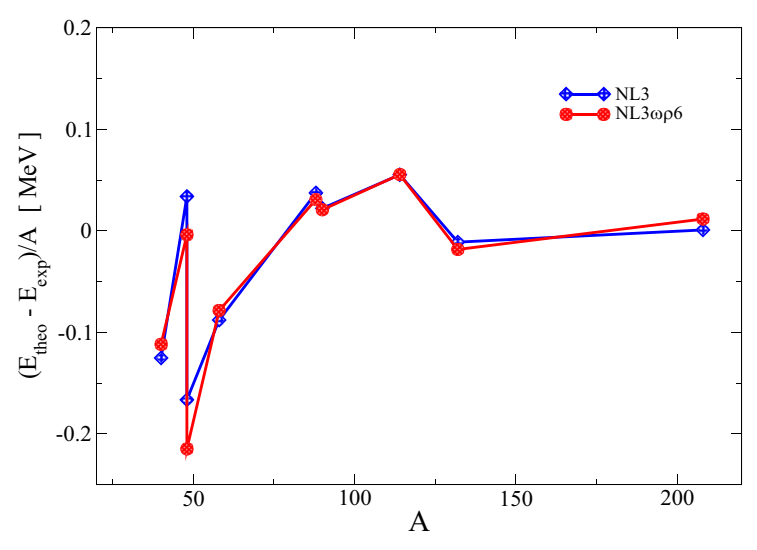

FIG. 3. Difference between theoretical and experimental energy per particle of some selected symmetric nuclei as a function of their mass number.

if the possibility of a neutron skin was included in our simple parametrization.

In spite of their very different properties concerning dripline nuclei, the NL3 and NL3 $\omega \rho 6$ functionals can be hardly discriminated from laboratory experiments. This is shown in Fig. 3, where we show the difference between theoretical and experimental binding energy per nucleon for the nuclei used in the fitting protocol. One can notice from the figure that both models reproduce the experimental nuclear masses at the same level of precision: the error in the binding energy per nucleon is not more than $200 \mathrm{keV}$ for the lighter nuclei, and much smaller for $A>100$ which will be the focus of the successive analysis.
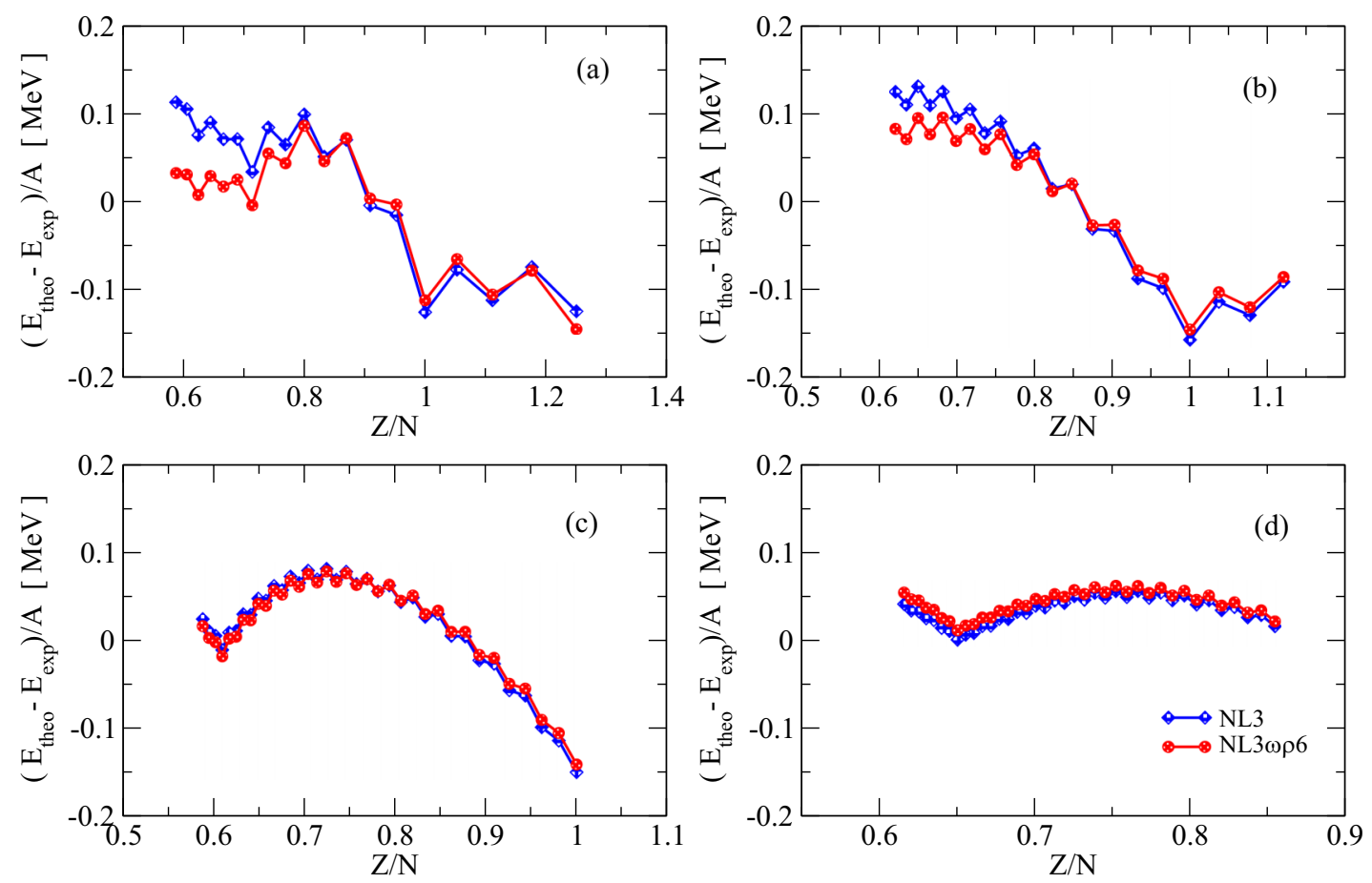

FIG. 4. Difference between theoretical and experimental energy per particle vs. proton to neutron ratio $Z / N$ for different $Z$ values (a) 20 , (b) 28 , (c) 50, and (d) 82, for the for NL3 and NL3 $\omega \rho 6$ models.

This improved model can in principle be applied both to study multifragmentation reactions and the properties of hot stellar matter at subsaturation densities. For this latter application we should let both the particle number and the volume to infinity such as to reach convergence of the observables in the thermodynamic limit. Such a study additionally allows nuclei which are not included in the fit. This can be appreciated from Fig. 4, which displays the difference between again observe that the two functionals perform equally well, except a better reproduction of light neutron rich nuclei for the softer symmetry energy case. This latter observation tends to favor a soft symmetry energy behavior. However, the evidence compelling because it is well known that liquid drop here, are not very realistic for light nuclei.

In particular, to improve the description for further applications to multifragmentation, where intermediate mass fragments are copiously produced, we plan to add curvature terms. Indeed it was recently shown [71] that the surface tenterms, leads to an extremely precise reproduction of complete extended Thomas-Fermi calculations for all proton fractions. We expect that such refinements, which are necessary for a better prediction power of data, might reduce the correlation between the bulk properties of matter and the experimental observables.

\section{B. Nuclear distributions at finite temperature}




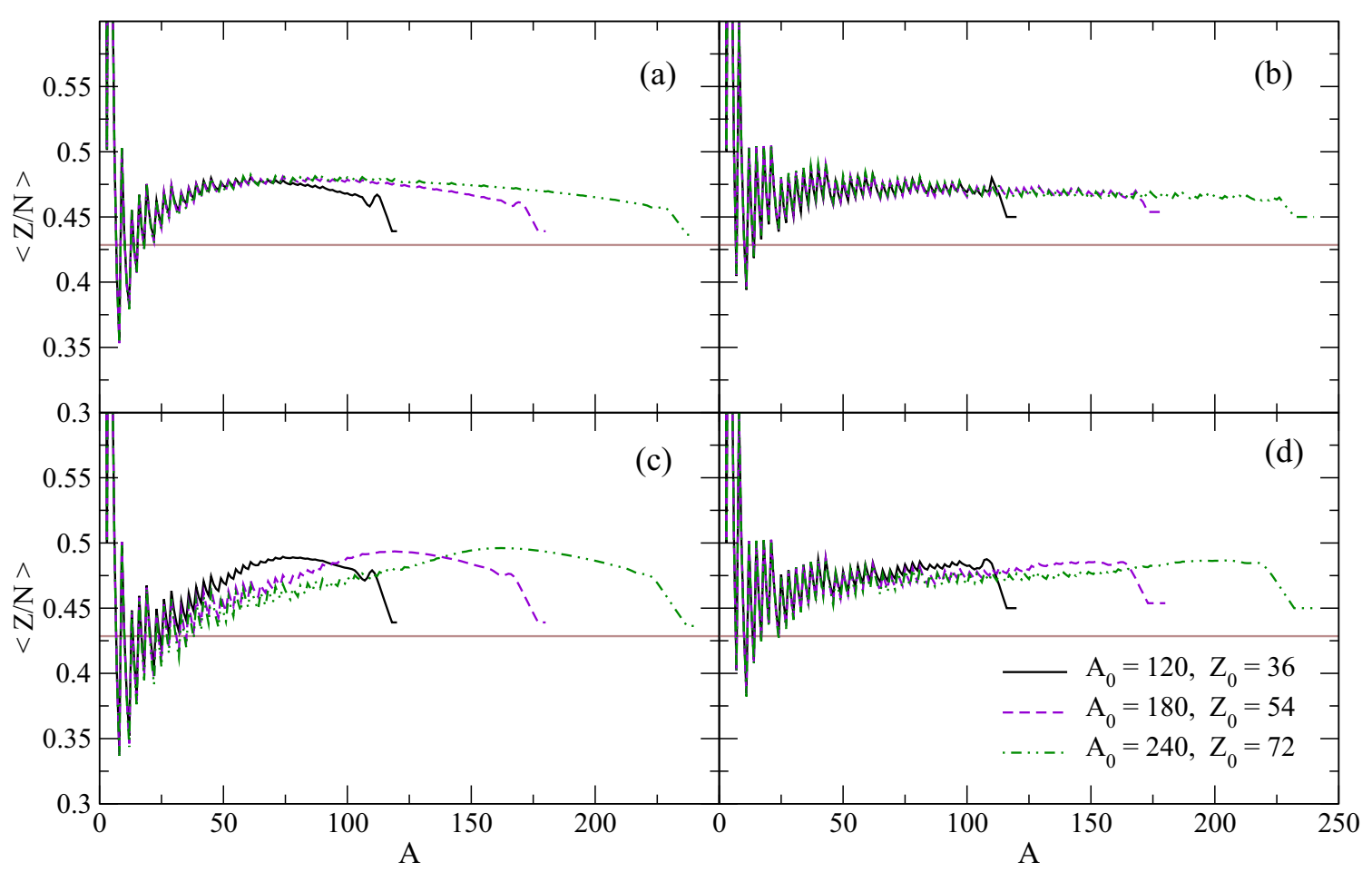

FIG. 5. $\langle Z / N\rangle$ of the clusters as a function of their size, with (lower panels) and without (upper panels) the Coulomb screening effect from the electron background. Left (right) panels: NL3 (NL3 $\omega \rho 6$ ). In all panels, $\mathrm{T}=4 \mathrm{MeV}, Y_{p}=0.3$, and $\rho / \rho_{0}=0.25$. The horizontal lines give the global $Z / N$ of the system. The total number of nucleons considered in each simulation is also given.

estimating the bias which is done when extrapolating laboratory results to the supernova context. For this first application of the model, we study the mass distributions and the fractionation properties of the system as measured by the average isotopic ratio of the fragments, and their dependence on the different environments, namely, (1) nuclei produced in multifragmentation reactions, i.e., at finite temperature but finite size and no lepton in the system, (2) hot nuclei along with the electrons, which will represent the supernova environnement in the thermodynamic limit.

The isotopic ratio is defined for each mass number $A$ as

$$
\left\langle\frac{Z}{N}\right\rangle(A)=\frac{\sum_{N} \frac{(A-N)}{N} n_{N, A-N}}{\sum_{N} n_{N, A-N}},
$$

where the sum runs from the proton to the neutron dripline, and $n_{N, Z}$ is given by Eq. (3). For all cases we consider a fixed thermodynamic condition typical for supernova matter studies, namely a temperature $\mathrm{T}=4 \mathrm{MeV}$, proton fraction $Y_{p}=$ 0.3 and density $\rho / \rho_{0}=0.25$. Changing this thermodynamic condition obviously changes our quantitative results, but not the qualitative observations on the fractionation properties in multifragmentation and stellar matter.

In Fig. 5 we present the variable $\langle Z / N\rangle$ of the fragments as a function of their mass number for case (1), namely, nuclei produced in multifragmentation reactions, and for case (2), fragments in the presence of leptons to maintain the global charge neutrality. For a uniform distribution of protons and neutrons, we would expect $\langle Z / N\rangle=Y_{p} /\left(1-Y_{p}\right) \approx 0.43$. The well-known fractionation phenomenon [72], that is the fact that the heavy fragments representing the liquid fraction of the system are more isospin symmetric than the global system, is clearly observed. The excess neutrons are mostly present in the form of free neutrons, with a free nucleon ratio $n_{0,1} / n_{1,0}$ of the order $\approx 10^{-3}\left(\approx 10^{-4}\right)$ without (with) the electron background, almost independent of the functional. For all considered cases, the isospin content of the fragments below $A=50$ is remarkably independent of the size of the source. Moreover, it is only very marginally affected by the presence of the electron background. This means that the results from fragmentation experiments can be extrapolated to supernova matter. The only difference stems from the endpoint of the curves, that reflects the presence of a heavy residue. Such residue is obviously limited by the finite size of the system in multifragmentation experiments, while it corresponds to the pasta structures in stellar matter.

The other common feature of the different calculations is the presence of staggering effects, which are understood as due to the fluctuating number of isobars existing between the driplines, and entering in the sums of Eq. (16). These fluctuations are very important for light nuclei, while the effect fades away with increasing mass number. This effect is more pronounced in the NL $3 \omega \rho 6$ model because of the lower number of isobars between the driplines; see Fig. 2.

Concerning the effect of the functional, we can also see that the stiffer model (NL3) leads to an increased fractionation effect, that is an increased difference between the minimum and maximum isospin as a function of the size, while the softer NL3 $\omega \rho 6$ model the isospin content is almost independent of the fragment size. 

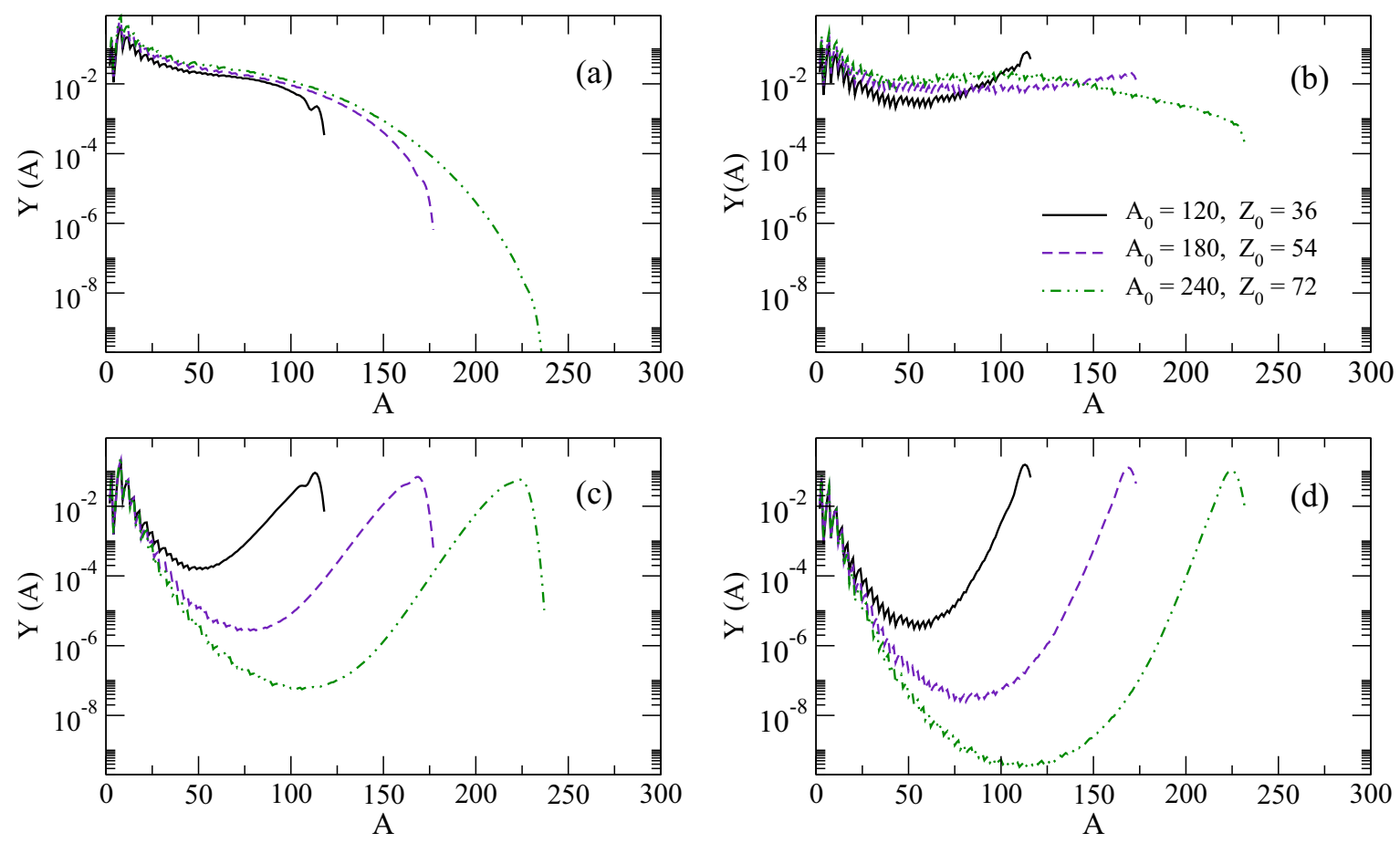

FIG. 6. Cluster multiplicity as a function of their size, with (lower panels) and without (upper panels) the Coulomb screening effect from the electron background. Left (right) panels: NL3 (NL3 $\omega \rho 6$ ). In all panels, $T=4 \mathrm{MeV}, Y_{p}=0.3$, and $\rho / \rho_{0}=0.25$. The total number of nucleons considered in each simulation is also given.

A complementary information is given in Fig. 6, which shows the abundancy of the different cluster sizes for the two models. We can see that in case of nuclei produced in multifragmentation reactions (upper panels), the distributions strongly depend in a non trivial way on the global size of the system. Very interestingly, the symmetry energy is seen to have an important effect on the global shape of the distributions. In the previous section we have seen that the symmetry properties of NL3 lead to a lower surface tension and an increased binding for neutron rich nuclei, and correspondingly more extended driplines. As a consequence, intermediate mass fragments are more copiously produced, filling the dip that we can observe in the case of NL3 $3 \rho 6$ between the very light fragments ("gas" component) and the heavy residue ("liquid" component). This is especially evident for the lowest system size (black lines in Fig. 6). The lower part of Fig. 6 corresponds to the case of matter neutralized by an electron background. In this case, the global pattern is the one expected from previous studies in subsaturation stellar matter [16-20], namely, the coexistence of a heavy component ("pasta" structure) with a nuclear gas given by a distribution of light constituents, exponentially decreasing with the cluster size. We can see that the distribution of the gas is independent of the total number of particles included in the calculation of the partition sum up to $A \approx 25$. Conversely, the size of the pasta structure is seen to linearly increase with the total system size. For the present thermodynamic conditions, the expected size of the pasta is of the order of $A \approx 1000$ [73], which explains why no convergence is observed on the high mass part of the curve. Even if we cannot estimate the average pasta size, we can see that a nice scaling is observed for the distributions, such that we can give convergent predictions for the mass fractions of the different components, as well as their average isotopic ratios. These fractions are reported in Table III. The gas component is defined as the exponential distribution, up to the cluster size $A_{\min }$ at which the multiplicity distribution of Fig. 6 shows a minimum. The sizes $A>A_{\text {min }}$ are considered to give the heavy pasta component.

We can observe that in this relatively high density condition, close to the core-crust transition in neutron stars, the heavy fragments exhaust most of the mass fraction $\left(X_{\text {heavy }}\right)$ as expected. The complementary mass fraction corresponding to the gas however is not only constituted by free nucleons $\left(X_{N}\right)$, but a nonnegligible fraction of the mass $\left(X_{\text {light }}\right)$ is bound in light clusters with $2 \leqslant A \leqslant A_{\min }$. This is especially true in the case of NL3, where the light clusters constitute almost $30 \%$ of the mass fraction of the gas. The presence of clusters of all sizes in the full thermodynamic equilibrium considered here, modifies drastically the isospin distribution with respect to the expectation in a two-phase coexistence scenario. Similar to a phase coexistence, the dilute (dense) phase given by free particles (heaviest cluster) is more (less) neutron rich than the global system, but the clusters of intermediate sizes exhibit a complex patters, and an important number of protons is bound in relatively small clusters with average mass number $\langle A\rangle<10$ (see Table III), rather than in the pasta structure as it would be the case in a simple two-component system. As a consequence, the average proton to neutron ratio of the gas component $\left(\langle Z / N\rangle_{\text {gas }}\right.$ in Table III) is three orders of magnitude higher than the free proton to neutron ratio $n_{01} / n_{10}$.

Finally, we look into the average $\langle Z / N\rangle_{\max }$, i.e., the maximum value of average $\langle Z / N\rangle$ of a fragment among all the 
TABLE III. Predictions from NL3 and NL3 $\omega \rho 6$ models of the matter composition at $T=4 \mathrm{MeV}, Y_{p}=0.3$, and $\rho / \rho_{0}=0.25$. Electron screening is included. See text for more details.

\begin{tabular}{|c|c|c|c|c|c|c|c|c|}
\hline Model & $X_{\text {heavy }}$ & $X_{\text {light }}$ & $X_{N}$ & $\langle A\rangle$ & $\langle Z / N\rangle_{\text {heavy }}$ & $\langle Z / N\rangle_{\text {gas }}$ & $\langle Z / N\rangle_{\text {light }}$ & $n_{0,1} / n_{1,0}$ \\
\hline NL3 $3 \omega \rho 6$ & 0.932 & 0.004 & 0.064 & 5.246 & 0.472 & 0.030 & 0.518 & $1.2 \times 10^{-4}$ \\
\hline
\end{tabular}

fragments produced in the system. As it can be appreciated from the comparison of Figs. 5 and 6 and is expected from general fractionation arguments, the most symmetric clusters correspond to the heaviest ones, and therefore $\langle Z / N\rangle_{\max }$ can be assimilated to the pasta structure of the single nucleus approximation. Indeed, even if the system size considered in Fig. 5 is not sufficient to lead to convergent results for the size of the pasta structure in supernova matter, its isospin content is remarkably independent of the size.

In Fig. 7, we have plotted the average $\langle Z / N\rangle_{\max }$ as a function of the number density $\rho / \rho_{0}$ for proton fraction $Y_{p}=0.3$ and 0.4 , using the two different functionals. The curves with different colors represent different environments for the nuclear system while the same temperature $\mathrm{T}=4 \mathrm{MeV}$ is kept for all the cases. One can see that though the isospin content

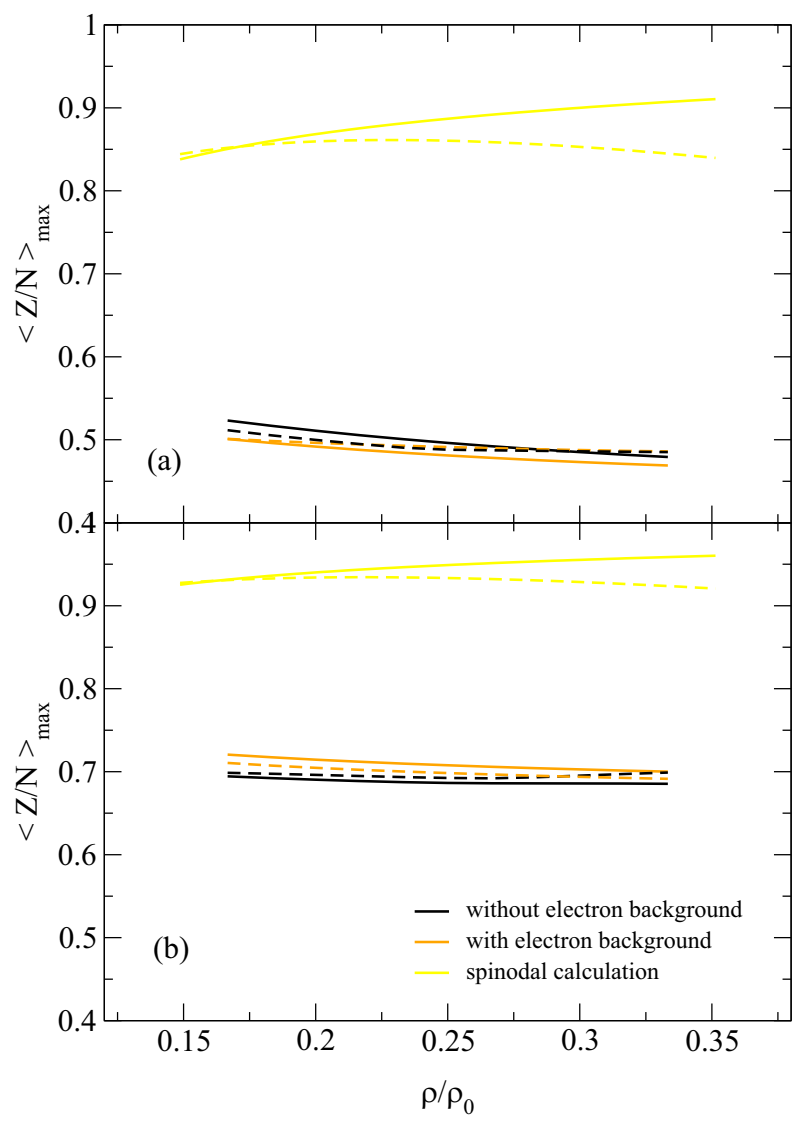

FIG. 7. $\langle\mathrm{Z} / \mathrm{N}\rangle_{\max }$ of the clusters as a function of density $\rho / \rho_{0}$ of the system for different cases. The temperature is fixed to $T=4 \mathrm{MeV}$ and $Y_{p}=0.3$ (upper panel) and $Y_{p}=0.4$ (lower panel). Results with the NL3 (NL3 $\omega \rho 6$ ) are given by full (dashed) lines. Results are also compared with the thermodynamic spinodal calculation. of the dense fraction obviously scales with the global proton fraction of the system, it is much less affected by the other parameters. The influence of the density is negligible, and the same is true for the effect of the functional. The only sizable effect is the one induced by Coulomb screening in the case of stellar matter, which leads as expected to slightly higher values for the charge content of the cluster.

We have also compared our results with the ratio $\delta \rho_{p}^{-} / \delta \rho_{n}^{-}$ giving the instability direction in a thermodynamic spinodal instability calculation [55]. We can see that the spinodal instability corresponds to a much higher fractionation than the one observed in the CTM model, and this is true for both models and all thermodynamic conditions. This might be surprising considering that the spinodal instability is thought to give a good estimation of the properties of clusterized matter at densities close to the transition to uniform matter, at least concerning the expected cluster sizes and transition densities $[16,55,73]$. A possible explanation could lie in the fact that the equilibrium isospin content is here compared to the prediction from the thermodynamical spinodal, which does not consider Coulomb and surface effects [74,75]. However, this does not seem a realistic explanation since it was shown for a large number of models including NL3 that the thermodynamic instability direction is very close to the one deduced from finite size fluctuations (compare for example Fig. 3 of Ref. [74] with Fig. 6 of Ref. [75]).

The explanation of this important discrepancy has rather to be looked for in the presence of a large number of clusters of different sizes in the full thermodynamic calculation of the finite temperature equilibrium. These clusters do not considerably reduce the mass fraction associated to the pasta structure, but they increase the proton fraction of the gas, thus modifying the fractionation properties.

\section{CONCLUSION}

In this work, an improved version of the canonical thermodynamical model (CTM) of nuclear multifragmentation) [33] was introduced, where the liquid-drop model-based cluster functional is replaced by an improved functional, with bulk properties fixed from microscopic RMF calculations and surface properties optimized on experimentally measured mass of spherical nuclei, as well as on the microscopically evaluated crust-core transition density of neutron stars. This improved CTM allows pinning down the influence of the nuclear functional, and particularly the symmetry energy, on nuclear multifragmentation observables. Moreover, pushing the calculations to the thermodynamical limit and introducing the effect of an electron background, we can determine the observables which are less perturbed by finite size effects and that can give reliable extrapolations to the properties of sub- 
saturation density warm stellar matter, such as it can be found in supernova matter in the cooling of proto-neutron stars.

For this first application of the model, we have concentrated on the mass distributions and isotopic properties of clusters produced in different environments. We have seen that the behavior of the symmetry energy impacts in an important way the mass distributions of neutron rich sources, which could be tested in multifragmentation experiments. The average $\langle Z / N\rangle$ of the clusters, as a function of the cluster size, is found to be nearly independent of the size of system for the different environments considered in our study, and to show a very weak dependence on the behavior of the symmetry energy. The well-known fractionation phenomenon, with the dense part of the system more isospin symmetric than the global system, is observed. However, the degree of fractionation is strongly reduced with respect to the expectations from a phase coexistence in a two-component system. We interpret this finding as an effect of the complex composition of matter at finite temperature, that cannot be reduced to a single heavy nucleus (or "pasta" structure) and a nucleon gas. Finally, the fraction of light clusters in supernova environnement as well as their average size shows a clear dependence on the symmetry energy, the stiffer model leading to an increased light cluster fraction and increased cluster size.

For a future work, it will be very interesting to quantitatively compare the size and isospin ratio of the heaviest cluster obtained including the full CTM cluster distribution, with a pasta calculation using the same energy functional, and compute the associated impurity factor which enters in the cooling dynamics of proto-neutron stars [58].

\section{ACKNOWLEDGMENT}

We acknowledge the support of IFCPAR/CEFIPRA under the Project No. 5804-3.
[1] T. Fischer, I. Sagert, G. Pagliara, M. Hempel, J. SchaffnerBielich, T. Rauscher, F.-K. Thielemann, R. Käppeli, G. Martínez-Pinedo, and M. Liebendörfer, Astrophys. J. Suppl. Ser. 194, 39 (2011).

[2] S. Heckel, P. P. Schneider, and A. Sedrakian, Phys. Rev. C 80, 015805 (2009).

[3] A. R. Raduta and F. Gulminelli, Phys. Rev. C 82, 065801 (2010).

[4] A. Botvina and I. Mishustin, Nucl. Phys. A 843, 98 (2010).

[5] M. Hempel and J. Schaffner-Bielich, Nucl. Phys. A 837, 210 (2010).

[6] S. Blinnikov, I. Panov, M. Rudzsky, and K. Sumiyoshi, Astron. Astrophys. 535, A37 (2011).

[7] S. Furusawa, S. Yamada, K. Sumiyoshi, and H. Suzuki, Astrophys. J. 738, 178 (2011).

[8] M. Hempel, T. Fischer, J. Schaffner-Bielich, and M. Liebendorfer, Astrophys. J. 748, 70 (2012).

[9] A. W. Steiner, M. Hempel, and T. Fischer, Astrophys. J. 774, 17 (2013).

[10] S. Furusawa, K. Sumiyoshi, S. Yamada, and H. Suzuki, Astrophys. J. 772, 95 (2013).

[11] F. Gulminelli and A. R. Raduta, Phys. Rev. C 92, 055803 (2015).

[12] S. Furusawa, K. Sumiyoshi, S. Yamada, and H. Suzuki, Nucl. Phys. A 957, 188 (2017).

[13] S. Furusawa, H. Togashi, H. Nagakura, K. Sumiyoshi, S. Yamada, H. Suzuki, and M. Takano, J. Phys. G 44, 094001 (2017),

[14] A. Raduta and F. Gulminelli, Nucl. Phys. A 983, 252 (2019).

[15] http://compose.obspm.fr/ [CompStar Online Supernovae Equations of State (CompOSE)].

[16] S. S. Avancini, C. C. Barros, D. P. Menezes, and C. Providência, Phys. Rev. C 82, 025808 (2010).

[17] M. Ferreira and C. Providência, Phys. Rev. C 85, 055811 (2012).

[18] H. Pais, S. Chiacchiera, and C. Providência, Phys. Rev. C 91, 055801 (2015).

[19] S. S. Avancini, M. Ferreira, H. Pais, C. Providência, and G. Röpke, Phys. Rev. C 95, 045804 (2017).
[20] H. Pais, F. Gulminelli, C. Providência, and G. Röpke, Phys. Rev. C 99, 055806 (2019).

[21] J. M. Lattimer and D. F. Swesty, Nucl. Phys. A 535, 331 (1991)

[22] T. Carreau, F. Gulminelli, and J. Margueron, Eur. Phys. J. A 55, 188 (2019).

[23] M. Hempel, K. Hagel, J. Natowitz, G. Röpke, and S. Typel, Phys. Rev. C 91, 045805 (2015).

[24] H. Pais, R. Bougault, F. Gulminelli, C. Providěncia, E. Bonnet, B. Borderie, A. Chbihi, J. D. Frankland, E. Galichet, D. Gruyer et al., Phys. Rev. Lett. 125, 012701 (2020).

[25] A. S. Botvina and I. N. Mishustin, Phys. Rev. C 72, 048801 (2005)

[26] N. Buyukcizmeci, A. S. Botvina, and I. N. Mishustin, Astrophys. J. 789, 33 (2014),

[27] T. Fischer, M. Hempel, I. Sagert, Y. Suwa, and J. SchaffnerBielich, Eur. Phys. J. A 50, 46 (2014).

[28] N. Buyukcizmeci, A. Botvina, I. Mishustin, R. Ogul, M. Hempel, J. Schaffner-Bielich, F.-K. Thielemann, S. Furusawa, K. Sumiyoshi, S. Yamada et al., Nucl. Phys. A 907, 13 (2013).

[29] G. Bertsch and S. D. Gupta, Phys. Rep. 160, 189 (1988).

[30] S. Mallik, S. Das Gupta, and G. Chaudhuri, Phys. Rev. C 89, 044614 (2014).

[31] A. Ono and H. Horiuchi, Prog. Part. Nucl. Phys. 53, 501 (2004).

[32] C. Hartnack, R. K. Puri, J. Aichelin, J. Konopka, S. A. Bass, H. Stoecker, and W. Greiner, Eur. Phys. J. A1, 151 (1998).

[33] C. B. Das, S. Das Gupta, W. G. Lynch, A. Z. Mekjian, and M. B. Tsang, Phys. Rep. 406, 1 (2005).

[34] S. Mallik and G. Chaudhuri, Phys. Lett. B718, 189 (2012).

[35] S. Mallik and G. Chaudhuri, Phys. Lett. B727, 282 (2013).

[36] G. Chaudhuri, F. Gulminelli, and S. Mallik, Phys. Lett. B724, 115 (2013).

[37] J. Bondorf, A. Botvina, A. Iljinov, I. Mishustin, and K. Sneppen, Phys. Rep. 257, 133 (1995).

[38] D. H. E. Gross, Phys. Rep. 279, 119 (1997).

[39] C. B. Das, S. Das Gupta, X. D. Liu, and M. B. Tsang, Phys. Rev. C 64, 044608 (2001).

[40] R. Nandi and S. Schramm, Phys. Rev. C 94, 025806 (2016).

[41] P. N. Alcain and C. O. Dorso, Phys. Rev. C 97, 015803 (2018). 
[42] A. S. Schneider, M. E. Caplan, D. K. Berry, and C. J. Horowitz, Phys. Rev. C 98, 055801 (2018).

[43] J. D. Walecka, Ann. Phys. (NY) 83, 491 (1974).

[44] J. Boguta and A. R. Bodmer, Nucl. Phys. A292, 413 (1977).

[45] B. D. Serot and J. D. Walecka, in Advances in Nuclear Physics, edited by J. W. Negele and E. Vogt (Plenum, New York, 1986), Vol. 16.

[46] G. A. Lalazissis, J. König, and P. Ring, Phys. Rev. C 55, 540 (1997).

[47] H. Muller and B. D. Serot, Nucl. Phys. A 606, 508 (1996).

[48] R. J. Furnstahl, C. E. Price, and G. E. Walker, Phys. Rev. C 36, 2590 (1987).

[49] Y. K. Gambhir, P. Ring, and A. Thimet, Ann. Phys. (NY) 198, 132 (1990).

[50] B. D. Serot and J. D. Walecka, Int. J. Mod. Phys. E 6, 515 (1997).

[51] S. S. Avancini, L. Brito, P. Chomaz, D. P. Menezes, and C. Providencia, Phys. Rev. C 74, 024317 (2006).

[52] C. Ducoin, J. Margueron, C. Providência, and I. Vidaña, Phys. Rev. C 83, 045810 (2011).

[53] H. Pais, A. Sulaksono, B. K. Agrawal, and C. Providência, Phys. Rev. C 93, 045802 (2016).

[54] H. Pais and C. Providência, Phys. Rev. C 94, 015808 (2016).

[55] N. Alam, H. Pais, C. Providência, and B. K. Agrawal, Phys. Rev. C 95, 055808 (2017).

[56] E. F. Brown and A. Cumming, Astrophys. J. 698, 1020 (2009).

[57] C. J. Horowitz, D. K. Berry, M. E. Caplan, T. Fischer, Z. Lin, W. G. Newton, E. O. Connor, and L. F. Roberts, arXiv:1611.10226 [astro-ph.HE] (2016).

[58] C. C. Barros, D. P. Menezes, and F. Gulminelli, Phys. Rev. C 101, 035211 (2020).

[59] S. Das Gupta and A. Z. Mekjian, Phys. Rev. C 57, 1361 (1998).
[60] P. Papakonstantinou, J. Margueron, F. Gulminelli, and A. R. Raduta, Phys. Rev. C 88, 045805 (2013).

[61] Z.-Y. Ma, J. Rong, B.-Q. Chen, Z.-Y. Zhu, and H.-Q. Song, Phys. Lett. B 604, 170 (2004).

[62] E. N. E. van Dalen, C. Fuchs, and A. Faessler, Phys. Rev. Lett. 95, 022302 (2005).

[63] B.-A. Li and X. Han, Phys. Lett. B 727, 276 (2013).

[64] T. von Egidy and D. Bucurescu, Phys. Rev. C 72, 044311 (2005).

[65] D. Ravenhall, C. Pethick, and J. Lattimer, Nucl. Phys. A 407, 571 (1983).

[66] C. P. Lorenz, D. G. Ravenhall, and C. J. Pathick, Phys. Rev. Lett. 70, 379 (1993).

[67] Y. Lim, C. H. Hyun, and C.-H. Lee, Int. J. Mod. Phys. E 26, 1750015 (2017).

[68] W. G. Newton, M. Gearheart, and B.-A. Li, Astrophys. J. Suppl. 204, 9 (2013).

[69] B. K. Agrawal, Phys. Rev. C 81, 034323 (2010).

[70] J. Carriere, C. Horowitz, and J. Piekarewicz, Astrophys. J. 593, 463 (2003).

[71] U. J. Furtado and F. Gulminelli, J. Phys. G: Nucl. Part. Phys. 48, 015102 (2020).

[72] V. Baran, M. Colonna, M. Di Toro, V. Greco, M. Zielinska-Pfabe, and H. H. Wolter, Nucl. Phys. A703, 603 (2002).

[73] S. S. Avancini, D. P. Menezes, M. D. Alloy, J. R. Marinelli, M. M. W. Moraes, and C. Providência, Phys. Rev. C 78, 015802 (2008).

[74] A. M. Santos, L. Brito, and C. Providência, Phys. Rev. C 77, 045805 (2008).

[75] C. Ducoin, C. Providência, A. M. Santos, L. Brito, and P. Chomaz, Phys. Rev. C 78, 055801 (2008). 